\section{(C) OPEN ACCESS}

\title{
Bacteria-free minicircle DNA system to generate integration-free CAR-T cells
}

\author{
Chen Cheng, ${ }_{1,2}^{1,2} \mathrm{Na}$ Tang, ${ }^{2}$ Jiaxin $\mathrm{Li}^{3}$ Shiwei Cao, ${ }^{2,4}$ Tongtong Zhang, ${ }^{2,4}$ Xiaofei Wei, ${ }^{5}$ \\ Haoyi Wang ${ }^{2,4}$
}

- Additional material is published online only. To view please visit the journal online (http://dx.doi.org/10.1136/ jmedgenet-2018-105405)

${ }^{1}$ School of Life Sciences, University of Science and Technology of China, Hefei, China

${ }^{2}$ State Key Laboratory of Stem Cell and Reproductive Biology, Institute of Zoology, The Chinese Academy of Sciences, Beijing, China

${ }^{3}$ Northwest Agriculture and Forestry University, Yangling, China

${ }^{4}$ University of the Chinese Academy of Sciences, Beijing, China

${ }^{5}$ Beijing Cord Blood Bank, Beijing, China

\section{Correspondence to}

Dr Haoyi Wang, University of the Chinese Academy of Sciences, Beijing 101408, China; wanghaoyi@ioz.ac.cn

CC and NT contributed equally.

Received 3 April 2018

Revised 5 June 2018

Accepted 18 June 2018

Published Online First 20 July 2018

Check for updates

(C) Author(s) (or their employer(s)) 2019. Re-use permitted under CC BY-NC. No commercial re-use. See rights and permissions. Published by BMJ.

To cite: Cheng C, Tang N, Li J, et al. J Med Genet 2019:56:10-17.

\section{ABSTRACT}

Background Chimeric antigen receptor T (CAR-T) cells engineered with lentiviral and retroviral vectors have been successfully applied to treat patients with B cell malignancy. However, viral integration in T cells has the potential risk of mutagenesis, and viral vector production demands effort and is costly. Using non-integrative episomal vector such as minicircle vector to generate integration-free CAR-T cells is an attractive option.

Methods and results We established a novel method to generate minicircle vector within a few hours using simple molecular biology techniques. Since no bacteria is involved, we named these vectors bacteria-free (BF) minicircle. In comparison with plasmids, BF minicircle vector enabled higher transgene expression and improved cell viability in human cell line, stem cells and primary $T$ cells. Using BF minicircle vector, we generated integration-free CAR-T cells, which eliminated cancer cells efficiently both in vitro and in vivo.

Conclusion BF minicircle vector will be useful in basic research as well as in clinical applications such as CAR-T and gene therapy. Although the transgene expression of minicircle vector lasts apparently shorter than that of insertional lentivirus, multiple rounds of $B F$ minicircle CAR-T cell infusion could eliminate cancer cells efficiently. On the other hand, a relatively shorter CAR-T cell persistence provides an opportunity to avoid serious side effects such as cytokine storm or on-target off-tumour toxicity.

\section{INTRODUCTION}

Chimeric antigen receptor $\mathrm{T}$ (CAR-T) cell therapy is one of the most promising treatments for cancer. In 2017, two CAR-T cell products were approved by the Food and Drug Administration (FDA) for the treatment of acute lymphoblastic leukaemia and advanced lymphomas, respectively. ${ }^{1}$ Currently, CAR-T cells in majority of the studies, including two FDA-approved products, are generated using lentiviral or retroviral vectors. ${ }^{12}$ Viral integration in $\mathrm{T}$ cells has the potential risk of mutagenesis, and the effort and cost of viral vector production and regulatory demands associated with clinical use make this virus-based treatment costly, therefore limiting its broad applications. ${ }^{3-5}$

Alternatively, non-integrative vectors are attractive options. A high level of transgene expression could be achieved shortly after DNA plasmid delivery into the target cells. However, the expression falls rapidly to a low level within a few days even if the DNA vectors are retained in these cells.
It has been reported that bacterial DNA linked to a mammalian expression cassette results in transcriptional silencing of episomal transgene. ${ }^{67}$ To address this issue, minicircle DNA vector devoid of bacterial backbone was developed. ${ }^{689}$ Compared with bacterial plasmids, minicircle episomal DNA vectors have more persistent and higher transgene expression in vivo, ${ }^{8} 10$ which make them attractive tools for gene therapy. Previously, different methods have been developed to produce minicircle vectors using specific producer plasmids and genetically modified bacterial strains, which usually take several days to finish. ${ }^{9}$ In addition, producing vectors using bacteria could lead to endotoxin contamination. $^{11}$

In this study, we established a novel method to produce minicircle vector within a few hours using simple molecular biology techniques, without using any bacteria strain. We name this vector bacteria-free (BF) minicircle. Compared with plasmids, BF minicircle vector enabled higher transgene expression and better cell viability in cell line, stem cells and primary $\mathrm{T}$ cells. In addition, we generated integration-free CAR-T cells using BF minicircle vector, and they eliminated cancer cells efficiently both in vitro and in vivo, with an efficacy comparable with CAR-T cells engineered with lentiviral vector.

\section{MATERIALS AND METHODS Production of BF minicircle vector}

To amplify target transgene, we designed 96 pairs of primers. The 5' end of each oligo contains BbsI site followed by 6 bp unique sequences. The PCR products digested by BbsI will have $4 \mathrm{bp}$ single strand overhang at both ends. The total possible combinausually take several days to finish.9 In addition, prod usually take several days to finish.9 In addition, prod tion of these $4 \mathrm{bp}$ overhang is $256\left(4^{4}\right)$, and since the overhang on one end of each PCR product needs to be compatible with that of the other end, the number of possible unique overhang pairs is 128 . Ninety-six pairs of primers were randomly selected from these 128 combinations, and the sequences of the primers used in this experiment are shown in online supplementary table S1.

Using these 96 pairs of primers, the target fragments (EF1a-019-2A-eGFP/CMVeGFP) were amplified from plasmids (Takara, PrimeSTAR HS DNA Polymerase, Cat: \#R010B) under the following conditions: $95^{\circ} \mathrm{C}$ for $5 \mathrm{~min} ; 35 \times\left(95^{\circ} \mathrm{C}\right.$ for $30 \mathrm{~s}, 58^{\circ} \mathrm{C}$ for $30 \mathrm{~s}, 68^{\circ} \mathrm{C}$ for $\left.10-40 \mathrm{~s}\right) ; 68^{\circ} \mathrm{C}$ for $2 \mathrm{~min}$; and hold at $4^{\circ} \mathrm{C}$. 
PCR products were pooled and purified using Qiagen QIAquick PCR Purification Kit (Cat No/ID: 28106). Restriction endonuclease BbsI was used to digest the PCR product (New England, Cat: \#R0539L). After purification (QIAquick PCR Purification Kit), the digested DNA fragments were ligated using T4 ligase (New England, Cat: \#M0202L) at $16^{\circ} \mathrm{C}$ for 2 hours, followed by T5 exonuclease (New England, Cat: \#M0363L) treatment at $37^{\circ} \mathrm{C}$ for 2 hours. The BF minicircle vectors were collected after a final round of DNA purification.

\section{Cell lines}

K562 (erythroleukaemia cell line) and Raji (Burkitt's lymphoma cell line) were purchased from American Type Culture Collection (ATCC). Raji-ffluc for bioluminescent imaging and K562CD19 cells were generated as previously described. ${ }^{12}$ All above cell lines were grown under standard conditions in RPMI 1640 medium (Thermo Fisher Scientific). Lentiviral producer cell lines 293T (ATCC-CRL3216) were maintained in Dulbecco's Modified Eagle Medium (DMEM) (Thermo Fisher Scientific). All mediums were supplemented with $10 \%(\mathrm{v} / \mathrm{v})$ fetal bovine serum, $100 \mathrm{U} / \mathrm{mL}$ penicillin and streptomycin, $2 \mathrm{mM} \mathrm{L}$-glutamine, and $1 \mathrm{mM}$ sodium pyruvate. All cell lines were grown at $37^{\circ} \mathrm{C}$ in a $5 \% \mathrm{CO}_{2}$ atmosphere.

\section{Primary human fresh umbilical cord blood-derived T cells}

Umbilical cord blood (UCB) units were obtained from Beijing Cord Blood Bank (Beijing, China) with informed consent from healthy volunteer donors. After Histopaque-1077 (Sigma-Aldrich) gradient separation, mononuclear cells were collected and T cells were isolated using the EasySep Human T Cell Enrichment Kit (Stemcell Technologies). T cells were stimulated with anti-CD3/CD28 Dynabeads (Thermo Fisher Scientific) at a bead to $\mathrm{T}$ cell ratio of $1: 1$ and cultured in X-Vivo 15 medium (Lonza), supplemented with $5 \%(\mathrm{v} / \mathrm{v})$ heat-inactivated fetal bovine serum, $2 \mathrm{mM}$ L-glutamine and $1 \mathrm{mM}$ sodium pyruvate in the presence of $300 \mathrm{IU} / \mathrm{mL}$ recombinant human interleukin (IL)-2 (all from Thermo Fisher Scientific).

\section{Primary human UCB-derived CD $34^{+}$haematopoietic stem cells and $\mathrm{H} 9$ cells}

UCB units were obtained from Beijing Cord Blood Bank with informed consent from healthy volunteer donors. After Histopaque-1077 (Sigma-Aldrich) gradient separation, mononuclear cells were collected and $\mathrm{CD} 34^{+}$haematopoietic stem cells (HSCs) were isolated using the EasySep Human Cord Blood CD34 Positive Selection Kit (Stemcell Technologies, \#17896). After selection, CD34 ${ }^{+}$cells were cultured in StemSpan H3000 (Stemcell Technologies, \#09800) added with expansion supplement (Stemcell Technologies, \#02691).

\section{Electroporation of $\mathrm{K} 562, \mathrm{CD} 34^{+} \mathrm{HSCs}$ and $\mathrm{H} 9$ cells}

$2 \times 10^{5} \mathrm{~K} 562$ cells were electroporated with $3 \mu \mathrm{g}$ PCR-eGFP or mini-eGFP or plasmid-eGFP by 4D-Nucleofector System $\mathrm{N}$ (Lonza) using the Amaxa SF Cell Line 4D-Nucleofector X Kit S (V4XC-2032) according to the manufacturer's instructions. The FF-120 program was used.

$5 \times 10^{4} \mathrm{CD} 34^{+}$HSCs were electroporated with $1 \mu \mathrm{g}$ minieGFP or plasmid-eGFP by 4D-Nucleofector System N (Lonza) using the P3 Primary Cell 4D-Nucleofector X Kit (V4XP3024, Lonza) according to the manufacturer's instructions. The EO-100 program was used.

$4 \times 10^{5} \mathrm{H} 9$ embryonic stem cells (ESCs) were electroporated with $1 \mu \mathrm{g}$ mini-eGFP or plasmid-eGFP by $4 \mathrm{D}-$ Nucleofector
System N (Lonza) using the P3 Primary Cell 4D-Nucleofector $\mathrm{X}$ Kit (V4XP-3024, Lonza) according to the manufacturer's instructions. The CB-150 program was used.

After electroporation, cells were resuspended with prewarmed cell medium and transferred into plate and incubated at $37^{\circ} \mathrm{C}$ in $5 \% \mathrm{CO}_{2}$. Cell viability and transfection efficiency were evaluated 2 days after electroporation by cell counting and fluorescence-activated cell sorting (FACS) analysis, respectively.

\section{Generation of lenti-019-CAR-T cells}

Freshly purified primary $\mathrm{T}$ cells were activated for 1 day and then transduced with lentiviral vectors harbouring a second-generation CD19 CAR. The structure of the CAR was previously described. ${ }^{12}$ The infection efficiency was evaluated 2 days after transduction by FACS analysis.

\section{Generation of mini-019-CAR-T cells}

T cells were activated for 3 days and then $1 \times 10^{6}$ cells were electroporated with $3 \mu \mathrm{g}$ mini-019-CAR DNA by 4D-Nucleofector System N (Lonza) using the P3 Primary Cell 4D-Nucleofector X Kit (V4XP-3024, Lonza) according to the manufacturer's instructions. The EO-115 program was used. After electroporation, cells were resuspended in $1 \mathrm{~mL}$ prewarmed $\mathrm{T}$ cell medium and transferred into 12 -well cell plate and incubated at $37^{\circ} \mathrm{C}$ in $5 \% \mathrm{CO}_{2}$. Cell viability and transfection efficiency were evaluated 2 days after electroporation by cell counting and FACS analysis, respectively.

\section{Luciferase-based CTL assay}

The cytotoxicity of CAR-T cell was assessed according to luciferase-based Cytotoxic T Lymphocyte (CTL) assay described previously. ${ }^{13}$ K562-luc and K562-CD19-luc cell lines were generated by transducing K562 and K562-CD19 cells with lentivirus expressing luciferase. Briefly, K562-luc and K562-CD19-luc cells were resuspended at $1 \times 10^{5}$ cells $/ \mathrm{mL}$ in $100 \mu \mathrm{L}$ RPMI 1640 medium and mixed with different ratios of effect cells (eg, 10:1, $5: 1$ and so on), with a total volume of $200 \mu \mathrm{L}$. After incubation at $37^{\circ} \mathrm{C}$ in $5 \% \mathrm{CO}_{2}$ for 16 hours, $10 \mu \mathrm{L}$ of substrate was added, and the luminescence was measured $5 \mathrm{~min}$ later. The results are reported as per cent killing based on the luciferase activity in the wells with tumour cells but no T cells (\% killing $=100-(($ RLU from well with effector and target cell coculture) / (relative light unit $(\mathrm{RLU})$ from well with target cells $) \times 100)$ ).

\section{Cytokine ELISA}

Cytokine release assays were performed by coculture of effector cells (T, mini-019-CAR-T) with target tumour cells (K562-CD19; K562) at a 1:1 ratio $\left(10^{4}\right.$ cells each) per well in duplicate in 96 -well plates in a final volume of $200 \mu \mathrm{L}$ complete RPMI 1640 medium. After 24 hours, supernatants were assayed for production of IL-2 and interferon (IFN)- $\gamma$ using ELISA Kit (BioLegend).

\section{Murine xenograft studies}

For establishing the Raji-ffluc tumour model, NOD-Prkdcscid Il2rgnull (NPG) mice 6-12 weeks old (VITALSTAR, Beijing, China) were injected with $2 \times 10^{5}$ Raji-ffluc cells via intraperitoneal injection on day 0 . Three days after injection, tumour engraftment was evaluated by serial biophotonic imaging using the NightOWL LB983 In Vivo Imaging System (Berthold Technologies). Mice with comparable tumour loads were segregated into different treatment groups and received different treatments: 


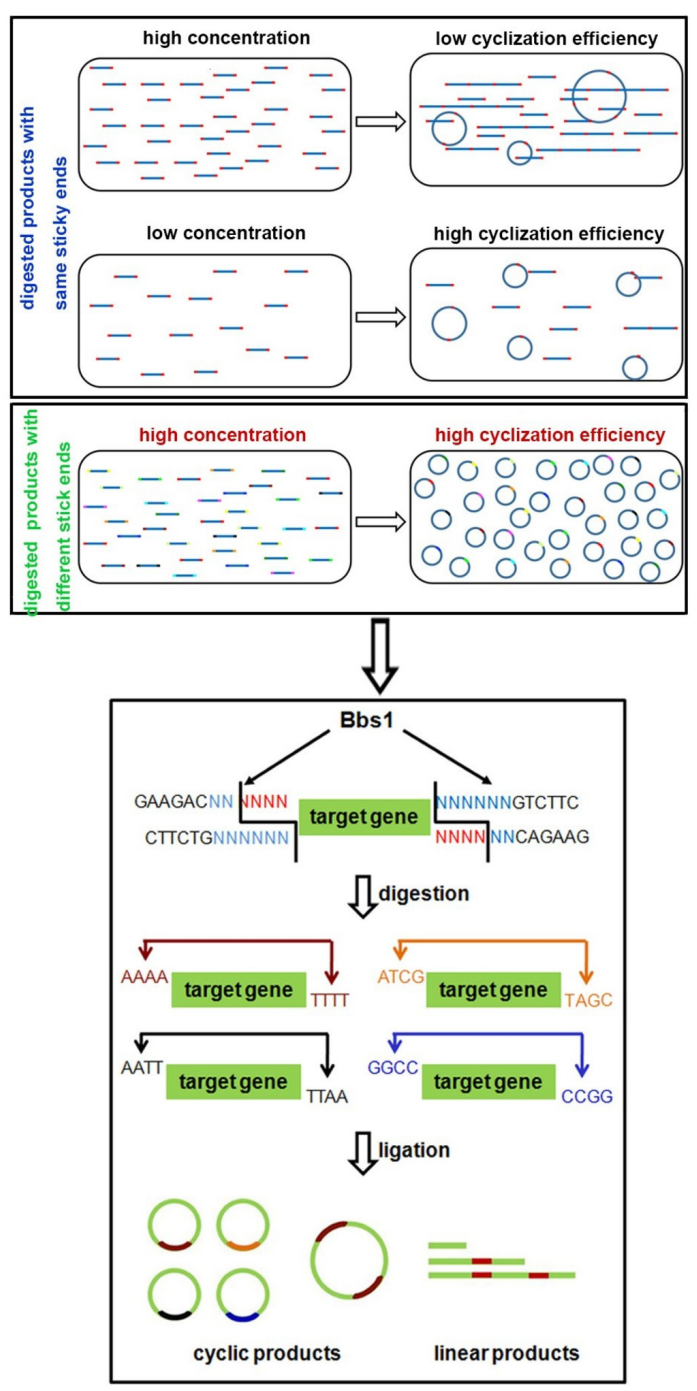

Figure 1 Schematic diagram of bacteria-free minicircle vector generation. The concentration of digested products affects the cyclisation efficiency of the minicircle molecule (upper panel) and the enzymatic digestion characterisation of Bbsl (lower panel).

phosphate-buffered saline (PBS) group, T cell group, lenti-019CAR-T cell group and mini-019-CAR-T cell group. Cells were administered at a dose of $5 \times 10^{6}$ cells/mouse via intraperitoneal injection. The tumour loads were evaluated 6 days after first treatment, and a second treatment was given with $5 \times 10^{6}$ cells/ mouse, and then tumour burden was evaluated every 7 days.

\section{Statistical analysis}

All statistical analyses were performed using GraphPad Prism V.6.0 as described in the figure legends. Data are expressed as mean \pm SD. Student's t-test was used for analyses between groups, and the difference was considered as $* * * \mathrm{p}<0.001$, $*$ p $<0.01$ and $* \mathrm{p}<0.05$.

\section{RESULTS}

\section{Generating BF minicircle vector}

To develop a novel method for minicircle vector generation without using bacteria, we exploited simple molecular biology techniques of enzyme digestion and self-ligation reactions. Linear DNA fragments with compatible overhangs can be ligated either in transforming concatemers or in cis forming circles (figure 1, upper panel). When the concentration of DNA fragments is high, they tend to ligate in trans. While decreasing the concentration leads to higher cyclisation efficiency, the low concentration makes it unpractical to achieve high yield. To achieve higher cyclisation efficiency under higher DNA concentration, we developed a multiplexed strategy (figure 1). Ninety-six pairs of primers were designed to amplify the transgene, with each pair composed of $20 \mathrm{bp}$ universal priming sequence, a $6 \mathrm{bp}$ unique sequence and a BbsI enzyme recognition site at the 5' end (online supplementary table S1). For PCR products generated using each pair of primers, BbsI digestion will generate compatible $4 \mathrm{bp}$ overhangs at each end (figure 1, lower panel). During ligation, PCR products produced using different primer pairs will not ligate with each other, as their ends are not compatible. Therefore, each DNA fragment will more likely ligate in cis forming circles.

To validate this design, we prepared $\mathrm{BF}$ minicircle vectors expressing eGFP or CAR recognising CD19 (019-CAR). We amplified transgene using 96 pairs of primers independently, using multichannel pipette and 96-well PCR plate. After PCR amplification, all products were pooled, digested with BbsI and ligated. On gel electrophoresis, multiple bands were observed. After getting rid of all linear molecules using T5 exonuclease, the remaining band ran lower than the linear PCR product on the gel, indicating this band was the minicircle molecule (figure 2A). Indeed, after we treated this product with restriction enzymes that only cut once within the minicircle molecule, it was linearised into the correct size (online supplementary figure S1). We quantified the efficiency of minicircle molecule formation under different DNA concentrations. When the sample was amplified using only one pair of primers, the cyclisation efficiencies decreased rapidly with the increase of DNA concentration. In comparison, the cyclisation efficiencies of transgene amplified using 96 pairs of primers remained at a high level even when the DNA fragment concentration was increased (figure $2 \mathrm{~B}$ and online supplementary figure S2). These results showed that we can generate minicircle vectors using a streamlined protocol within a few hours (figure 3).

\section{BF minicircle vectors enable robust transgene expression in cell line}

We transfected BF minicircle vectors, linear PCR products and plasmids encoding eGFP to K562 cells by electroporation, and found that the cell viability in BF minicircle group was higher than that in plasmid group (online supplementary figure S3A). Compared with the rapid decline in PCR product group and plasmid group, both the percentage of eGFP-positive cells and the mean fluorescence intensity (MFI) of eGFP remained high in cells transfected with BF minicircle vector in the first 8 days, although they fell gradually thereafter (online supplementary figure S3B,C). From these data, we conclude that, compared with plasmid and linear DNA fragment, BF minicircle vector has the advantage of maintaining high cell viability and transgene expression level.

\section{BF minicircle vector enables robust transgene expression in human CD34 ${ }^{+}$HSCs and ESCs}

Next, we characterised the performance of BF minicircle vector in CD $34{ }^{+}$HSCs isolated from umbilical blood. We found the MFI of eGFP in BF minicircle vector samples was much higher than in samples transfected with plasmid, while the eGFP-positive rates in both samples were similarly high (figure 4A,B). Meanwhile, the cell viability in $\mathrm{BF}$ minicircle vector electroporation group 


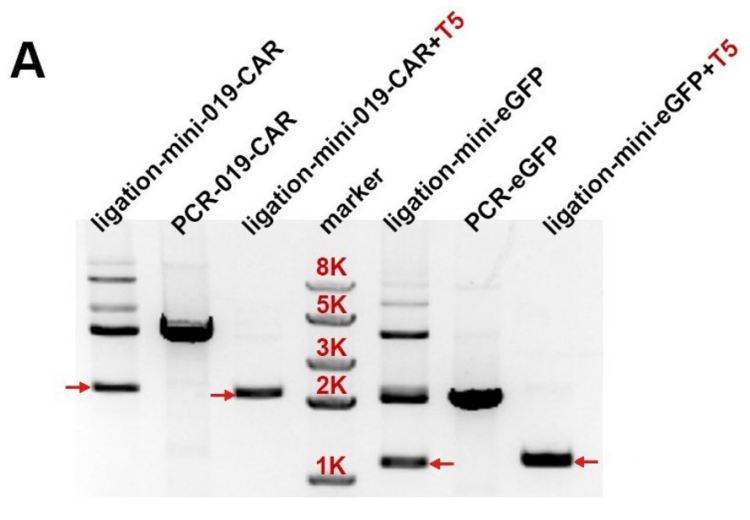

B

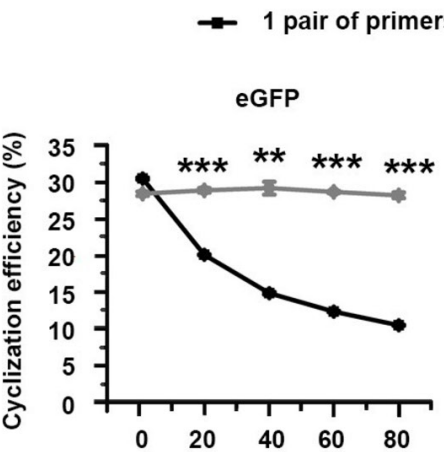

96 pairs of primers

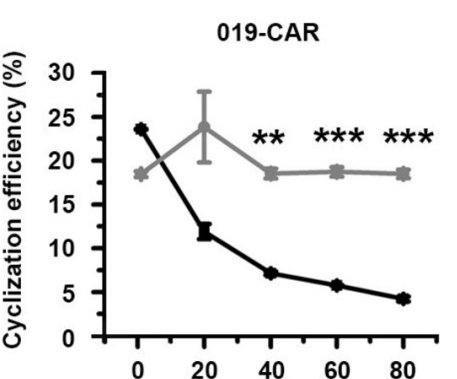

Figure 2 Cyclisation of 019-CAR and eGFP transgene fragments. (A) Agarose gel electrophoresis of PCR fragments, and minicircle ligation products before and after T5 exonuclease treatment. (B) Cyclisation efficiencies of eGFP and 019-CAR transgene amplified with 1 pair of primers or 96 pairs of primers. Unpaired multiple two-tailed t-test. The asterisks in (B) indicate significant differences between 96 pairs of primers group with 1 pair of primers in each different DNA concentration: ${ }^{* *} p<0.01,{ }^{* * *} p<0.001$.

was significantly higher than the plasmid group (figure 4C). To characterise the differentiation potential of electroporated CD $34^{+}$HSCs, colony forming unit analysis was used. The electroporated cells formed colonies successfully, and erythroid, granulocyte, macrophage and megakaryocyte colonies were found in each group, suggesting that the stemness of HSCs was not affected (figure 4D).

To evaluate the performance of $\mathrm{BF}$ minicircle vector in human ESCs, we electroporated BF minicircle vector and plasmid encoding eGFP into H9 cells. ${ }^{14}$ The eGFP expression and cell survival of BF minicircle vector-transfected cells were much better than that of plasmid (figure 4E-H). The $\mathrm{H} 9$ cells expressed OCT4 and NANOG uniformly, indicating that these cells were pluripotent (data not shown). All these data demonstrated that

\section{Figure 3}

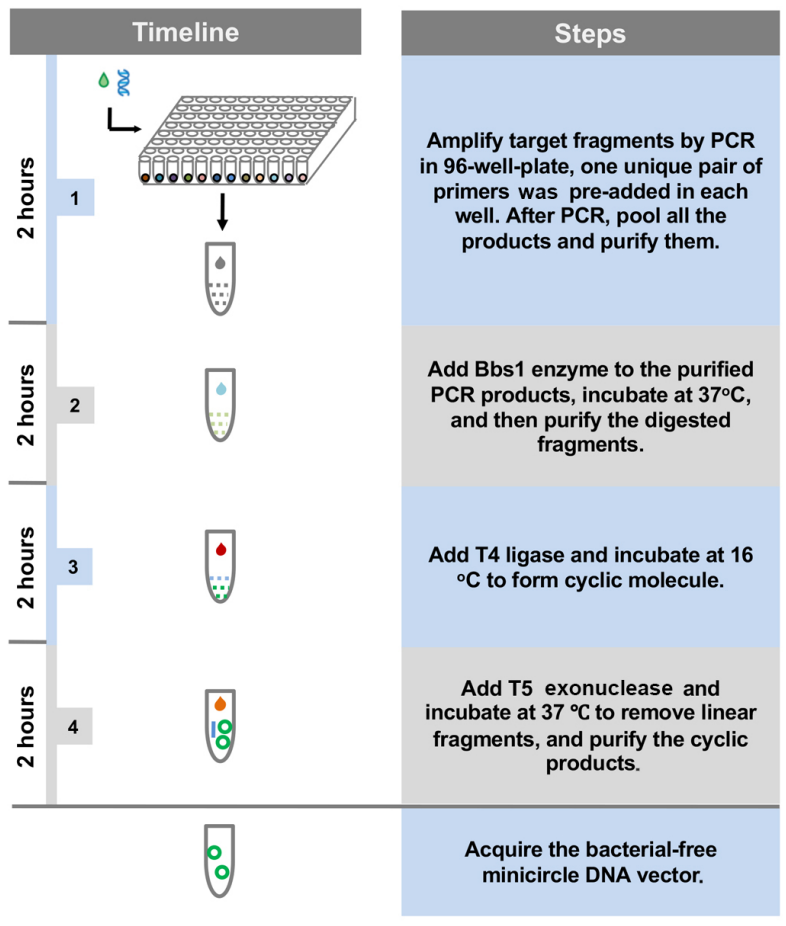

Figure 3 Flow chart of bacteria-free minicircle vector generation.
BF minicircle vector performed better than plasmid in stem cells, as evidenced by enhanced transgene expression level and cell viability.

\section{Generating CAR-T cells using BF minicircle vectors}

Next we tested whether we could generate transgene integration-free CAR-T cells using BF minicircle vector encoding 019-CAR and mediate antigen-specific cancer killing. We first compared the transgene expression levels of 019-CAR in primary $\mathrm{CD}^{+} \mathrm{T}$ cells transfected with $\mathrm{BF}$ minicircle vector and plasmid. $\mathrm{T}$ cells electroporated with plasmid survived poorly, while the cells in BF minicircle vector group showed a much higher viability and proliferating rate (figure $5 \mathrm{~A}, \mathrm{~B}$ ). BF minicircle vector enabled efficient CAR expression in primary $\mathrm{T}$ cells (figure $5 \mathrm{C}$ ). These BF minicircle CAR-T cells (mini-019-CAR-T) secreted high levels of IL-2 and IFN- $\gamma$ after incubation with CD19-positive tumour cells (figure 5D), and killed them at different effector to target ratios with an efficiency similar to CAR-T cells generated using lentivirus (lenti-019-CAR-T) (figure 5E).

As a control, we also generated 019-CAR minicircle vector (Kit-minicircle-019-CAR) using a commercially available minicircle vector producing kit (System Bioscience, USA), which is based on engineered bacteria strain and donor vector. ${ }^{9}$ Although we followed the manufacturer's instruction rigorously and tried multiple times, the Kit-minicircle vector product was often contaminated with plasmid and genomic DNA to a certain extent (online supplementary figure S4A). After electroporation, the T cell survival rate in Kit-minicircle-019-CAR group was much lower than that in the BF mini-019-CAR group (online supplementary figure S4B), which might be due to the low purity of Kit-minicircle-019-CAR. We could not acquire enough Kit-minicircle-019-CAR-T cells for further function analysis.

\section{BF minicircle CAR-T cells eradicated tumour efficiently in murine xenograft model}

Next we compared the tumour alleviating capability of BF mini019-CAR-T and lenti-019-CAR-T cells in a murine xenograft model. NPG mice were inoculated with Raji-luci cells intraperitoneally. Three days later, these mice were divided into four groups with similar tumour burden. T cells and CAR-T cells engineered with BF minicircle vector or lentiviral vector were administrated intraperitoneally on day 3 and day 9 (figure 6A). Tumour burden was monitored weekly by in vivo luciferase imaging. We 


\section{Figure 4}

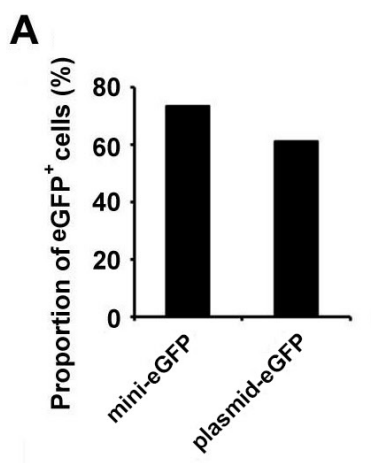

E

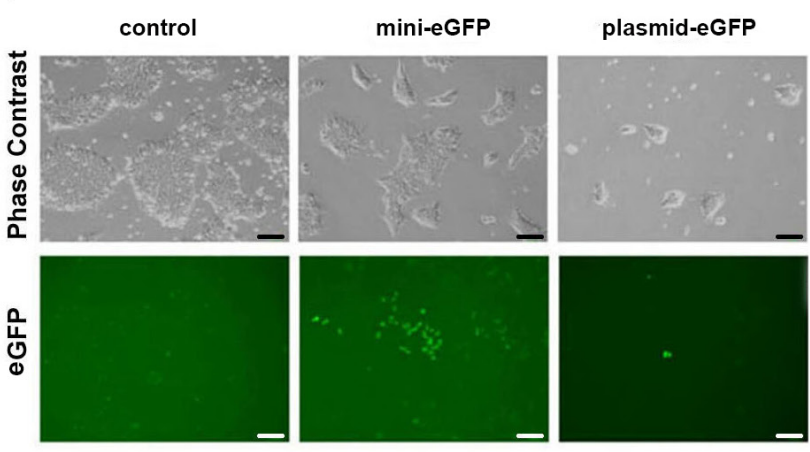

B

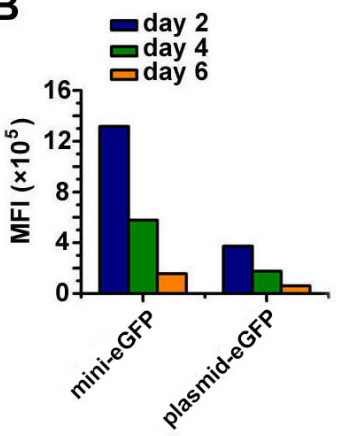

C

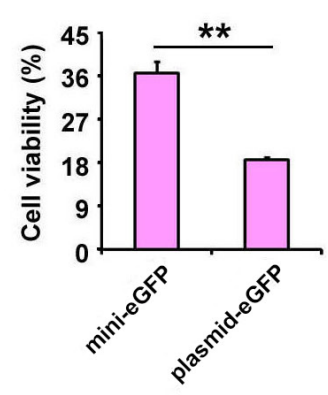

D

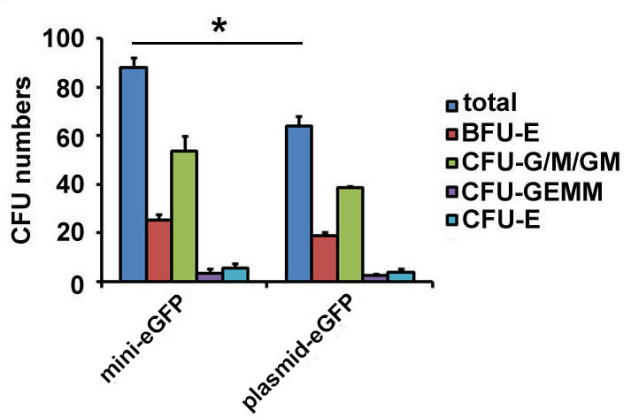

F

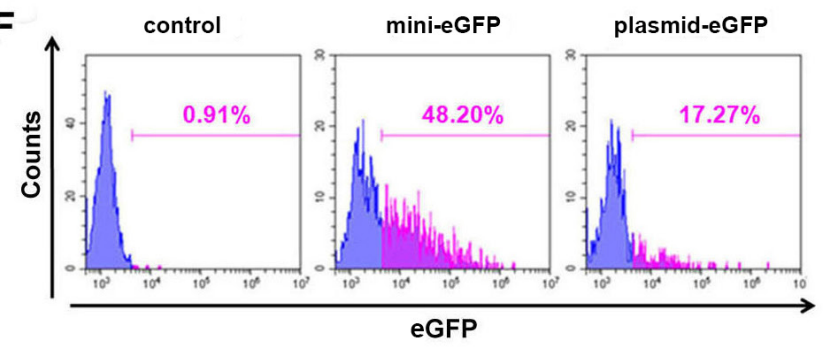

G

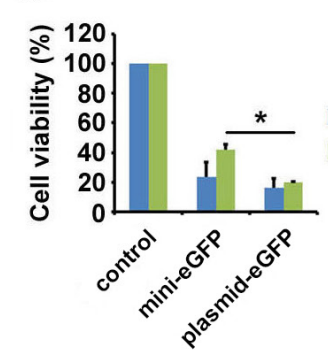

Figure 4 BF minicircle vector electroporation in CD34 $4^{+} \mathrm{HSCs}$ and hESCs. (A) Proportion of eGFP-positive cells in CD34 ${ }^{+} \mathrm{HSCs}$ transfected with BF minicircle vector and plasmid encoding eGFP 48 hours after electroporation. (B) The MFI of eGFP-positive cells in days 2, 4 and 6 after mini-eGFP and plasmid-eGFP electroporation. (C) Cell viability of CD34 ${ }^{+} \mathrm{HSC}$ transfected with BF minicircle vector and plasmid encoding eGFP 48 hours after electroporation. (D) Quantification of total CFU numbers and the numbers of BFU-E, CFU-G/M/GM, CFU-GEMM and CFU-E of CD34 ${ }^{+}$HSCs transfected with BF minicircle vector and plasmid encoding eGFP. (E) Typical images of H9 hESCs transfected with BF minicircle vector or plasmid encoding eGFP, 48 hours after electroporation. Scale bar $=100 \mu \mathrm{m}$. (F) FACS analysis of eGFP-positive cells in $\mathrm{H9}$ transfected with BF minicircle vector or plasmid encoding eGFP, 48 hours after electroporation. (G) Cell viability of $\mathrm{H} 9$ transfected with BF minicircle vector or plasmid encoding eGFP, 2 days and 6 days after electroporation. (H) Proportion of eGFP-positive cells in H9 transfected with BF minicircle vector or plasmid encoding eGFP, 2, 4 and 6 days after electroporation. (A-D) Data of CD34 ${ }^{+}$HSCs from one donor and more than three donor samples were detected. Unpaired multiple two-tailed t-test. The asterisks indicate significant differences between the mini-eGFP group and the plasmid-eGFP group: ${ }^{*} p<0.05,{ }^{* *} p<0.01$. BF, bacteria-free; CFU, colony forming unit; eGFP, enhanced green fluorescent protein; FACS, fluorescence-activated cell sorting; HSC, haematopoietic stem cell; MFI, mean fluorescence intensity.

found the tumour burden decreased significantly in mini-019CAR-T-group and lenti-019-CAR-T-group, compared with that in the T cell group and PBS group (figure 6B,C). Impressively, the efficacy of mini-019-CAR-T is similar to lenti-019-CAR-T, indicating that CAR-T cells engineered with BF minicircle vector have robust tumour alleviating ability.

\section{DISCUSSION}

Minicircle DNA molecule without any bacterial components is a valuable tool for basic research and gene therapy. Previous methods generating minicircle vector are time-consuming and require specific producer plasmids and bacteria strains. When we tried to produce minicircle vector using a commercial kit based on engineered bacterial strain, we could not produce minicircle vectors with high purity, and it took a few days to finish the procedure. Although this result could be due to our lack of experience with this kit, it showed the efforts required to use this system.

The method we established in this study allows for fast generation of minicircle vectors without using any bacteria culture, therefore eliminating any potential contamination from bacteria. The procedure to prepare BF minicircle can be summarised into five simple steps: (1) adding universal priming sequence to the transgene fragment by PCR; (2) PCR amplification of targeted gene fragment using 96 pairs of primers; (3) digestion of pooled PCR products by BbsI; (4) ligation of digested products by T4 ligase; and (5) removing linear products by T5 exonuclease (figure 3). With this method, we could acquire BF minicircle within 10 hours. 


\section{Figure 5}

A

mini-019-CAR

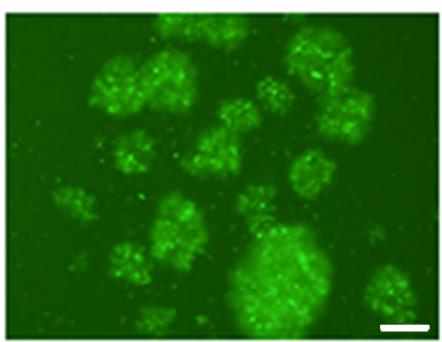

plasmid-019-CAR

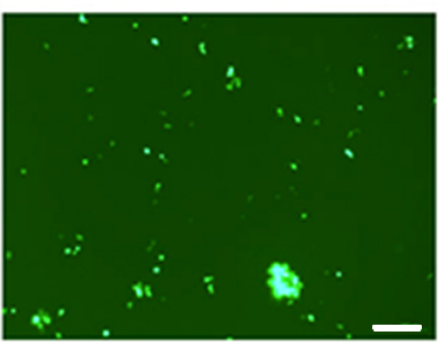

B

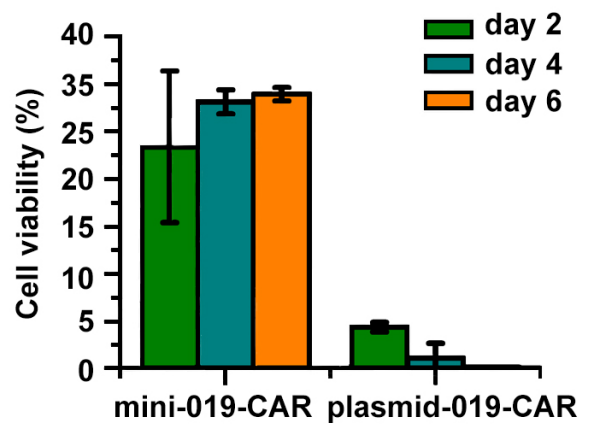

C

donor \#1

E

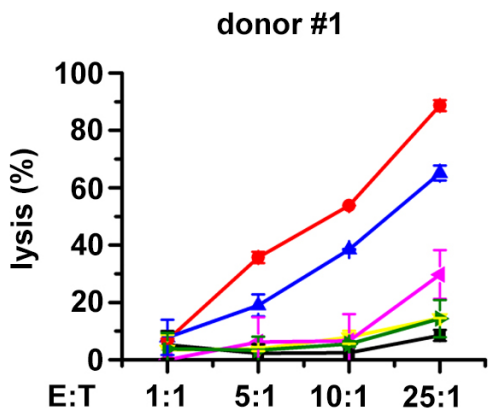

donor \#2

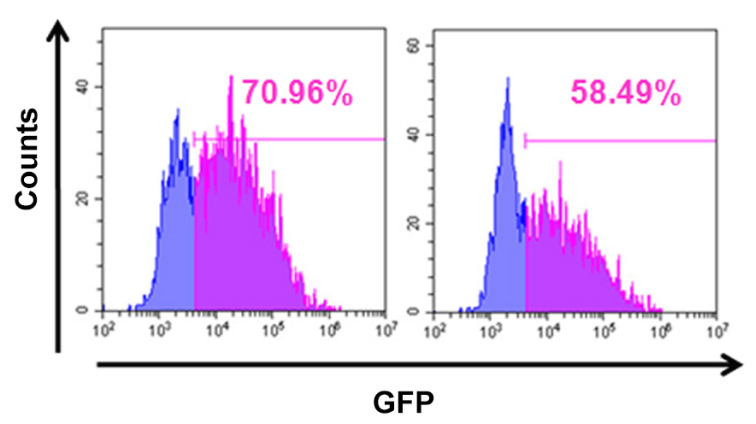

D

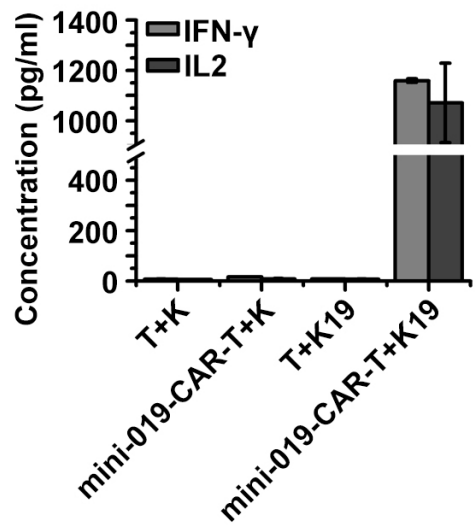

donor \#1

donor \#2

Figure 5 Antitumour effects of mini-019-CAR-T cells in vitro. (A) T cells transfected with mini-019-CAR and plasmid-019-CAR. Photos were taken 48 hours after electroporation. Scale bar $=100 \mu \mathrm{m}$. (B) Cell viability of T cells transfected with BF minicircle vector and plasmid on days 2, 4 and 6 . (C) Expression level of 019-CAR in T cells transfected with BF minicircle vector, analysed by FACS analysis. T cells were isolated from umbilical blood from two different donors. (D) Concentrations of IFN- $\gamma$ and IL-2 released by mini-019-CAR-T cells 24 hours after incubation with tumour cells. T cells were isolated from umbilical blood from two different donors. (E) Tumour cell lysis ability of 019-CAR-T cells prepared with BF minicircle vector (mini-019-CAR-T) or lentiviral vector (lenti-019-CAR-T) in different effector to target (E:T) ratios. T cells were isolated from umbilical blood from two different donors. T, T cells; K, K562 cell line; K19, K562 cell line overexpressing CD19. BF, bacteria-free; CART-T, chimeric antigen receptor T; GFP, green fluorescent protein; IFN- $\gamma$, interferon- $\gamma_{i}$ IL-2, interleukin-2.

Although minicircle vector has been used to deliver Sleeping Beauty system to generate CAR-T cells, ${ }^{3}$ our study is the first to show that integration-free CAR-T cells generated using BF minicircle vector eradicated cancer cells efficiently both in vitro and in vivo. Adoptive transfer of engineered $\mathrm{T}$ cells has shown remarkable responses in patients with diseases such as cancer and viral infections. ${ }^{15}$ Transgene encoding T cells receptor (TCR) or CAR targeting tumour antigens are usually cloned in viral vectors. On viral transduction, transgenes expressing TCR or CAR are integrated into $\mathrm{T}$ cell genome. While transgene integration allows for constitutive expression, it also poses risk of insertional mutagenesis. In addition, the costly clinical-grade virus production is one 


\section{Figure 6}

A

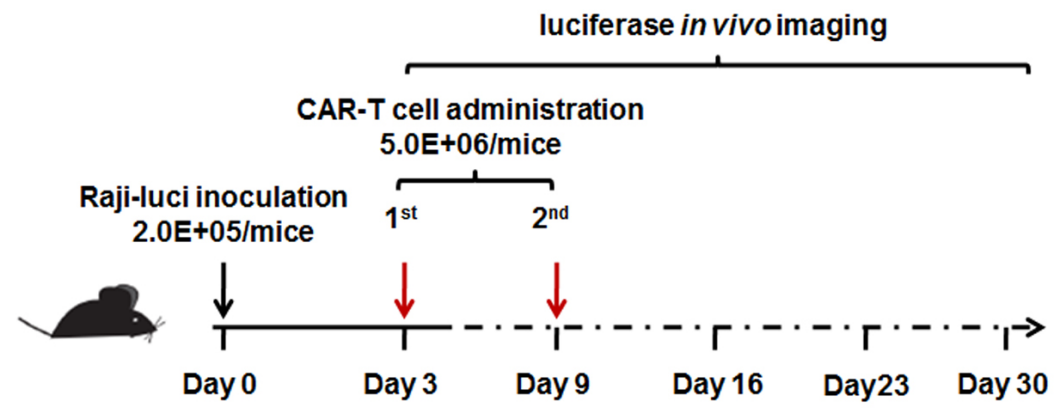

C

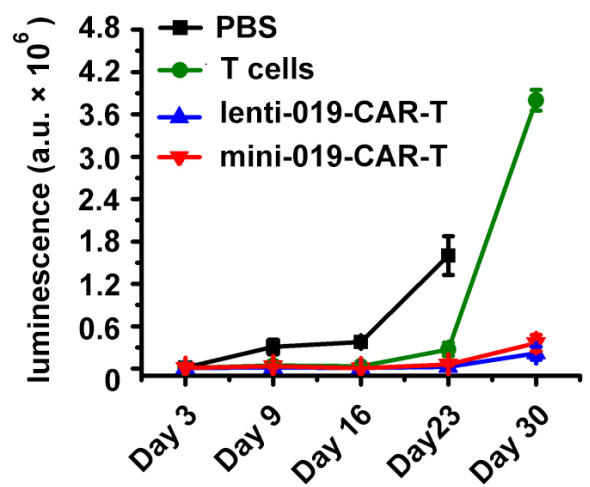

B

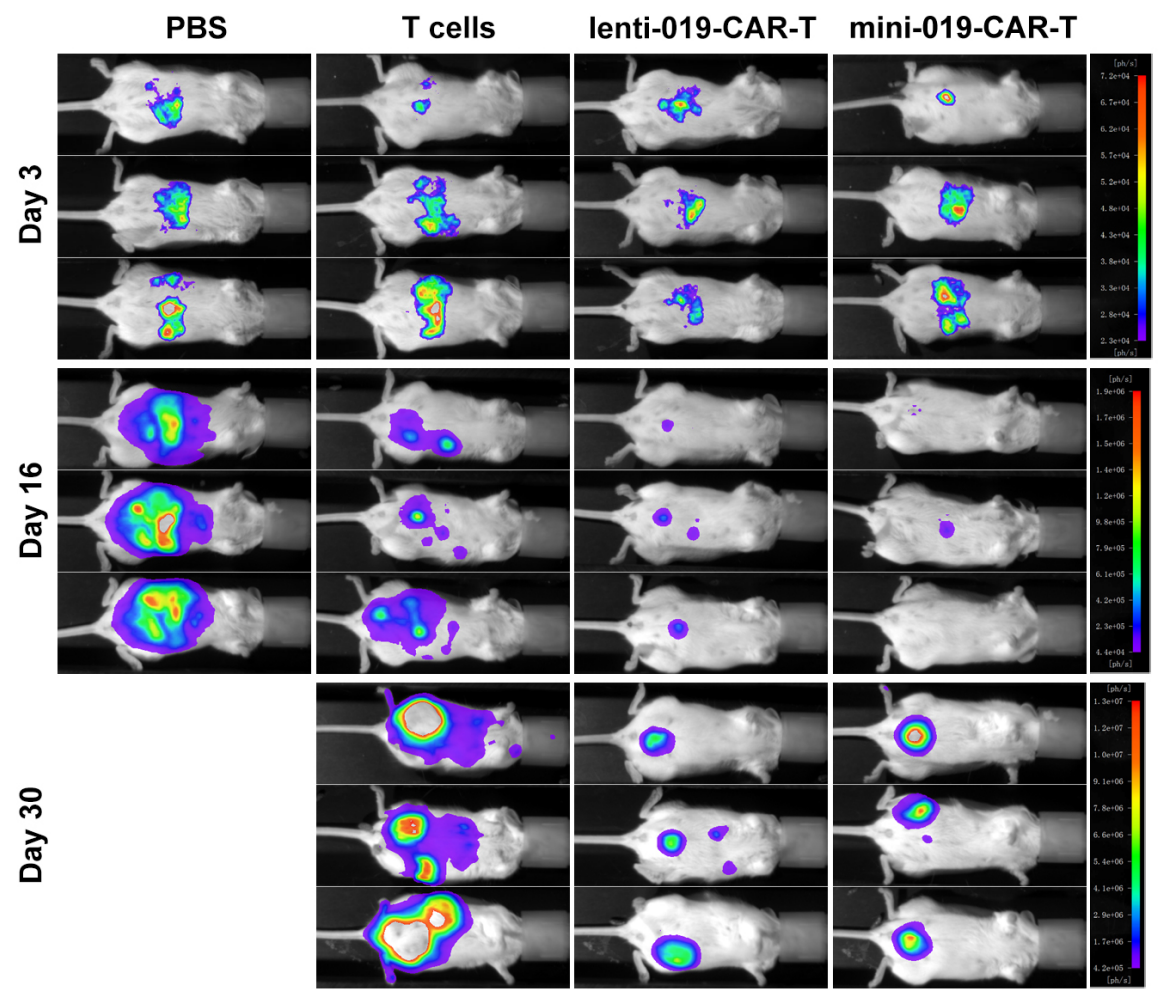

Figure 6 Antitumour effects of mini-019-CAR-T cells in vivo. (A) Experimental design of in vivo function test of CAR-T cells engineered with BF minicircle vector and lentiviral vectors. (B) Bioluminescent imaging result of NPG mice bearing Raji-luciferase tumour treated with T cells, lenti-019-CAR-T cells, mini019-CAR-T cells or the same volume of PBS on days 3,16 and 30 ( $n=3)$. (C) Quantification of luminescence at different time points. BF, bacteria-free; CAR-T, chimeric antigen receptor T; NPG, NOD-Prkdcscid II2rgnull.

of the major reasons for the high cost of clinical application. The recently FDA-approved CTL019 therapy ('Kymriah') has a price tag of $\$ 475000$. Our data suggest that transgene integration-free mini-019-CAR-T cells could be an attractive option for clinical applications. Although the length of transgene expression of BF minicircle vector is apparently shorter than lentivirus integrated in the genome, multiple rounds of infusion should be able to eliminate cancer cells efficiently, especially when a humanised CAR is used. On the other hand, a relatively shorter CAR-T persistence provides an opportunity to avoid serious side effects such as cytokine storm or on-target off-tumour toxicity. ${ }^{16}$
Using current protocol, the efficiency of mini-eGFP generation was about $30 \%-40 \%$ and mini-019-CAR was $20 \%-30 \%$. Further optimisations, such as simplifying the preparation procedure, reducing product loss in each step and increasing cyclisation efficiencies of the DNA fragments, will be needed to achieve better yield of BF minicircle vector. Another caveat of our method is that the minicircle vector we generated is a mixture of 96 different molecules, each containing a unique 4 bp sequence at the ligation junction. Considering these $4 \mathrm{bp}$ sequences are not being expressed and unlikely to affect transgene expression, this should not lead to any functional variations. 
In conclusion, we established a simple and fast method to prepare minicircle vectors without using bacteria and generated functional mini-019-CAR-T cells. Compared with retrovirus or lentivirus, $\mathrm{BF}$ minicircle vector has the potential to improve safety and lower the production costs of CAR-T therapy and gene therapy.

Acknowledgements We would like to thank Junning Wei and Yi Yang (Beijing Cord Blood Bank) for their help in preparing the cord blood samples. We would also like to thank Lei Shi (Beijing Cord Blood Bank) for her help with CFU analysis.

Contributors CC, NT and HW designed the experiments. CC, NT, JL, SC, TZ and XW performed the experiments. CC, NT and HW wrote the manuscript. All authors discussed and interpreted the results.

Funding This study was funded by the Strategic Priority Research Program of the Chinese Academy of Sciences (grant no: XDA01010409, XDA16010205), State 863 project (grant no: 2015AA020307), National Natural Science Foundation of China (10.13039/501100001809) (grant nos: 31471215 and 81773269), and the National Key Research and Development Program of China (no 2016YFA0101402). HW is supported by the 'Young Thousand Talent Project'.

Competing interests None declared.

Patient consent Obtained.

Provenance and peer review Not commissioned; externally peer reviewed.

Open access This is an open access article distributed in accordance with the Creative Commons Attribution Non Commercial (CC BY-NC 4.0) license, which permits others to distribute, remix, adapt, build upon this work non-commercially, and license their derivative works on different terms, provided the original work is properly cited, appropriate credit is given, any changes made indicated, and the use is non-commercial. See:http://creativecommons.org/licenses/by-nc/4.0/.

\section{REFERENCES}

1 Liu Y, Zhao C, Gao L, Yang H, He R, Gao C. Considerations for clinical review of cellular therapy products: perspectives of the China food and drug administration center for drug evaluation. Hum Gene Ther 2018;29:121-7.
2 Gill S, Maus MV, Porter DL. Chimeric antigen receptor T cell therapy: 25years in the making. Blood Rev 2016;30:157-67.

3 Monjezi R, Miskey C, Gogishvili T, Schleef M, Schmeer M, Einsele H, Ivics Z, Hudecek M. Enhanced CAR T-cell engineering using non-viral Sleeping Beauty transposition from minicircle vectors. Leukemia 2017;31:186-94.

4 Fischer A. Gene therapy: Myth or reality? C R Biol 2016;339:314-8.

5 Branca MA. Gene therapy: cursed or inching towards credibility? Nat Biotechnol 2005:23:519-21.

6 Nafissi N, Slavcev R. Construction and characterization of an in-vivo linear covalently closed DNA vector production system. Microb Cell Fact 2012;11:154.

7 Nicol F, Wong M, MacLaughlin FC, Perrard J, Wilson E, Nordstrom JL, Smith LC. Poly-L-glutamate, an anionic polymer, enhances transgene expression for plasmids delivered by intramuscular injection with in vivo electroporation. Gene Ther 2002;9:1351-8.

8 Chen ZY, He CY, Ehrhardt A, Kay MA. Minicircle DNA vectors devoid of bacterial DNA result in persistent and high-level transgene expression in vivo. Mol Ther 2003;8:495-500.

9 Kay MA, He CY, Chen ZY. A robust system for production of minicircle DNA vectors. Nat Biotechnol 2010;28:1287-9.

10 Huang M, Chen Z, Hu S, Jia F, Li Z, Hoyt G, Robbins RC, Kay MA, Wu JC, Jc W. Novel minicircle vector for gene therapy in murine myocardial infarction. Circulation 2009;120(11 Suppl):S230-S237.

11 Gordillo GM, Xia D, Mullins AN, Bergese SD, Orosz CG. Gene therapy in transplantation: pathological consequences of unavoidable plasmid contamination with lipopolysaccharide. Transpl Immunol 1999;7:83-94.

12 Liu X, Zhang Y, Cheng C, Cheng AW, Zhang X, Li N, Xia C, Wei X, Liu X, Wang H. CRISPR-Cas9-mediated multiplex gene editing in CAR-T cells. Cell Res 2017:27:154-7.

13 Ren J, Liu X, Fang C, Jiang S, June CH, Zhao Y. Multiplex genome editing to generate universal CAR T Cells Resistant to PD1 Inhibition. Clin Cancer Res 2017;23:2255-66.

14 Thomson JA, Itskovitz-Eldor J, Shapiro SS, Waknitz MA, Swiergiel JJ, Marshall VS, Jones JM. Embryonic stem cell lines derived from human blastocysts. Science 1998:282:1145-7.

15 Khalil DN, Smith EL, Brentjens RJ, Wolchok JD. The future of cancer treatment: immunomodulation, CARs and combination immunotherapy. Nat Rev Clin Oncol 2016;13:394.

16 Johnson LA, June $\mathrm{CH}$. Driving gene-engineered T cell immunotherapy of cancer. Cell Res 2017;27:38-58. 